\title{
Sistema de adquisición de datos por puerto paralelo para monitoreo y control de temperatura en ambientes cerrados
}

\author{
System of acquisition of data for parallel port in monitoreo \\ and control of temperature in closed atmospheres
}

\author{
Roberto Francisco Fernández Jara*
}

http://dx.doi.org/10.21503/CienciayDesarrollo.2010.v11.07

\section{RESUMEN}

Las personas que viven en áreas urbanas pasan entre el 80 y el $90 \%$ de su tiempo realizando actividades sedentarias en espacios interiores, tanto durante el trabajo como durante el tiempo de ocio.

Este hecho ha llevado a la creación de ambientes interiores más confortables y homogéneos que los exteriores, sujetos a condiciones climáticas variables.

Para ello, ha sido necesario acondicionar el aire de estos espacios, calentándolo en invierno y enfriándolo en verano. Para que el sistema de acondicionamiento fuera eficaz y rentable, había que controlar el aire que entraba en los edificios desde el exterior, cuyas características térmicas eran contrarias a las deseadas. Ello se tradujo en edificios cada vez más herméticos y en un control más riguroso de la cantidad de aire exterior utilizada para renovar las atmósferas interiores más viciadas.

Otros escenarios más inciertos son por ej. los de un invernadero, en el cual la temperatura sí es crítica, ya que de ello depende la vida de las especies en su interior, sea el caso que una especie como la Orquídea, y esta sea la especie de cultivo; esta depende directamente de la temperatura del ambiente para su desarrollo y crecimiento; entonces, se hace necesario el control de la temperatura del invernadero, y de manera automática, para el ahorro en costo de cantidad de personal y efectividad de corrección en los cambios drásticos de temperatura.

Palabras Clave: circuito integrado, $T T L$, transductor, BJT, tiristor, PCB, EPP, $A D C$.

\section{ABSTRACT}

People living in urban areas spend between 80 and $90 \%$ of their time doing sedentary activities indoors, both at work and during leisure time.

This has led to the creation of more comfortable indoor and outdoor homogeneous than subject to variable weather conditions.

It has therefore been necessary to put the air from these spaces, heating in winter and cool in summer. For the conditioning system to be effective and profitable, you had to control the air entering buildings from outside, whose thermal characteristics are contrary to those desired. This resulted in increasingly airtight buildings and tighter control on the amount of outside air used to renovate the interior atmospheres more flawed.

Other scenarios are critical eg. those of a greenhouse in which temperature is critical if, as it depends on the life of the species within them, the case that a species such as orchid, and this is the kind of crop, this depends directly on the room temperature for its development and growth, it becomes necessary to control the greenhouse temperature, and automatically, for cost savings in staff numbers and effectiveness of correction in drastic temperature changes.

Key words: intrgrated circuit, TTL, transducer, BJT, tiristor, PCB, EPP, $A D C$.

\footnotetext{
* Alumno de la Escuela Académico-Profesional de Ingeniería de Sistemas e Informática, Filial Arequipa.
} 


\section{INTRODUCCIÓN}

La agricultura siempre se ha visto afectada en demasía por factores externos que no dependen del agricultor: la lluvia (tanto por exceso como por defecto), la temperatura (tanto demasiado alta como demasiado baja), el viento, el granizo, y un largo etcétera que llevaría mucho tiempo enumerar.

Desde el principio, el agricultor ha intentado controlar en mayor o menor medida las adversidades de la naturaleza, usando distintas técnicas: riego, fertilización, cultivo en invernadero.

Pese a esto sigue habiendo demasiada dependencia del clima en la agricultura, que hace que en muchos casos los agricultores sufran importantes reveses económicos, debidos a circunstancias climátológicas que no pueden controlar.

No podemos cruzarnos de brazos ante esta eventualidad. Existen actualmente modernas técnicas para el control climático, que hacen que seamos cada vez menos dependientes de las circunstancias climáticas, y que nuestra rentabilidad dependa exclusivamente de nuestro esfuerzo.

Los factores climáticos que afectan a la agricultura son:

- Luz.

- Temperatura.

- Humedad del aire.

- Concentración de $\mathrm{CO}_{2}$.

- Viento.

Cuanto más control tengamos sobre dichos factores, más éxito y seguridad tendrá el agricultor en su actividad. Los sistemas de control climático garantizan un perfecto control de estos factores climáticos.
Mediante el control climático, no sólo aumenta la seguridad del agricultor, se incrementa la calidad y la producción, y por ello la rentabilidad de éste. Se puede adelantar la siembra y, con ello, la recolección, saliendo al mercado en momentos en que los precios son más favorables; se puede producir en épocas en que antes no se producía y, si se hacía, era a costa de reducir la producción y la calidad, por ejemplo, en pleno verano.

\section{Problema de la investigación}

No se cuenta con una herramienta que permita monitorear y controlar la temperatura de un ambiente cerrado en forma automática; de bajo costo y consumo de recursos.

\section{Objetivo de la investigación}

Controlar en formaautomáticala temperatura de un punto de área más critico en un ambiente cerrado, mediante la implementación de un sistema de adquisición de datos para el registro y monitoreo de temperatura mediante una PC (Pentium III) y por su puerto paralelo.

\section{Justificación e importancia}

\section{Justificación}

Con la construcción del proyecto se logrará contar con una herramienta para el monitoreo de ambientes cerrados, invernaderos que necesiten ser monitoreados en cuanto al grado de templanza, así como aquellos que su desempeño se vea afectado por la variación de su temperatura.

\section{Importancia}

\section{La importancia que tiene para el investigador}

El poder aplicar y adquirir nuevos conocimientos y técnicas en cuanto a conversión de señales analógico a digitales, procesamiento de señales mediante dispositivos electrónicos, 
control y acceso a puerto paralelo mediante DLL y otras técnicas, así como principios de sistemas de control automático de lazo cerrado (retroalimentación)

\section{La importancia que tiene para la Universidad}

La importancia para la Universidad radica en contar con una herramienta para el monitoreo continuo de temperatura, el cual ayuda a la detección de equipos con sobrecalentamiento y rendimiento afectado por la temperatura; asimismo, la detección del componente que es el directamente relacionado con el mal funcionamiento y su desempeño tras varias horas de funcionamiento.

La importancia que tiene para el jefe de investigación agraria

La importancia radica en la posibilidad de monitorear y detectar las variaciones y la estabilidad de la temperatura requerida en un ambiente cerrado (invernadero), para investigación agraria y cultivo de especies en invernaderos.

\section{MATERIAL Y MÉTODO}

\section{Descripción de la solución}

\section{Generalidades}

El sistema consta de 2 partes fundamentales, software el cual consta del aplicativo que gobernará nuestro sistema, el cual registrará los eventos en una BD.

Y el hardware, que consta de los sensores de temperatura, el circuito ADC que convertirá los datos analógicos en digitales para el ingreso al computador.

Cabe destacar que también tendremos los llamados "actuadores", los cuales son los encargados de ejecutar las acciones, que en nuestro caso sería activar y desactivar la calefacción y la refrigeración.

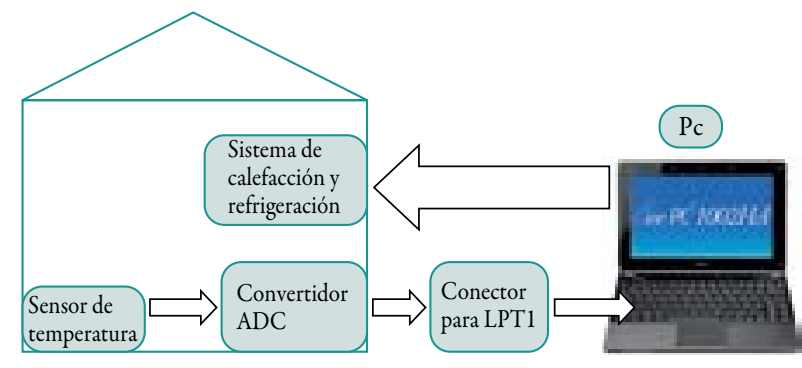

Fuente: Elaboración propia.

Figura 1. Diagrama conceptual del sistema de control automático.

\section{Requisitos funcionales}

Un requisito funcional define el comportamiento interno del software: cálculos, detalles técnicos, manipulación de datos y otras funcionalidades específicas que muestran cómo los casos de uso serán llevados a la práctica. Son complementados por los requisitos no funcionales, que se enfocan, en cambio, en el diseño o la implementación.

Para nuestro caso se definieron los siguientes:

- R01: Mantener la temperatura. El sistema debe mantener en forma automática la temperatura del ambiente cerrado (invernadero).

- R02: Registrar activación actuadores. El sistema debe registrar todos los cambios de temperatura que activaron los actuadores.

- R03: Mostrar gráfico T\%/t. El sistema debe mostrar una curva de Temperatura vs. Tiempo en tiempo real.

- R04: Estado de sensores. El sistema debe informar acerca de la funcionalidad y estado de los sensores de temperatura.

- R05: Control de Usuario. El sistema deberá identificará los usuarios del sistema y se deberá hacer que estos empleen los módulos que le permitan sus responsabilidades.

- R06: Ingresos de parámetros. El administrador es el que ingresa los parámetros de temperatura, límite inferior y superior para el control de la temperatura del ambiente cerrado (invernadero). 
Análisis de casos de uso

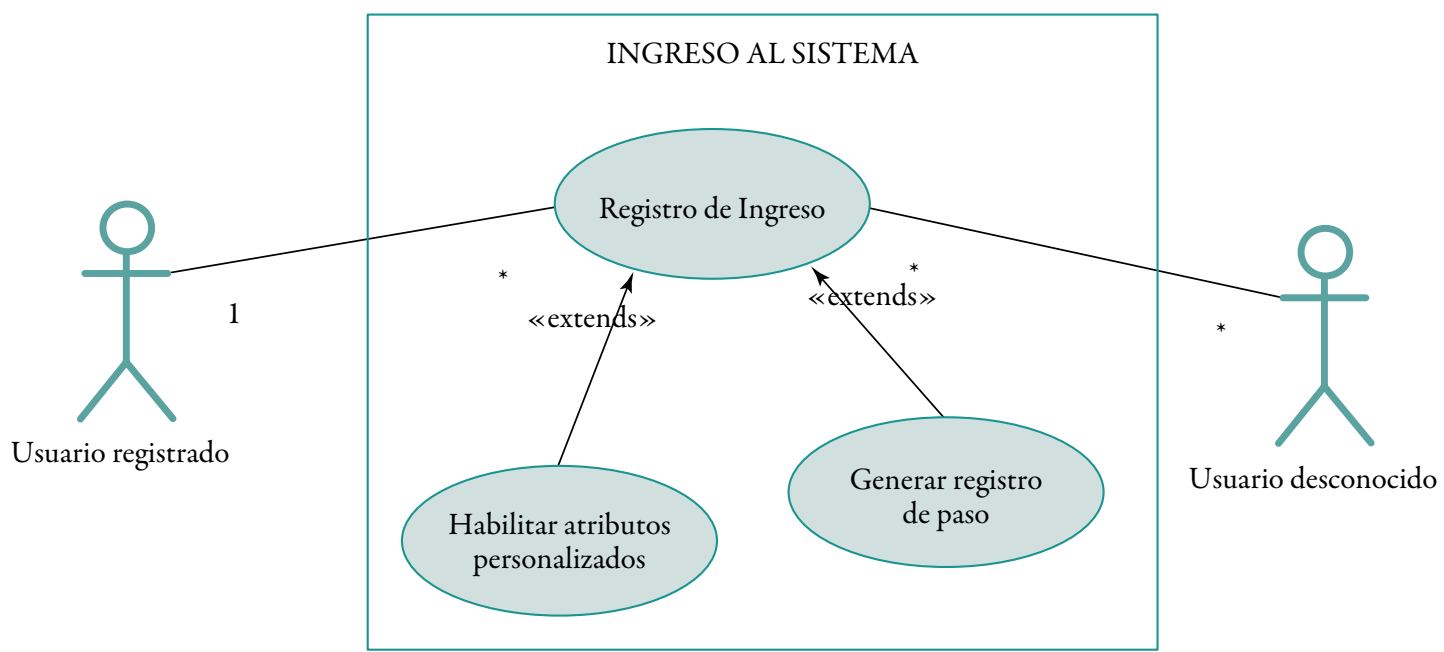

Figura 1. Diagrama de caso de uso: ingreso al sistema. DCU-001.

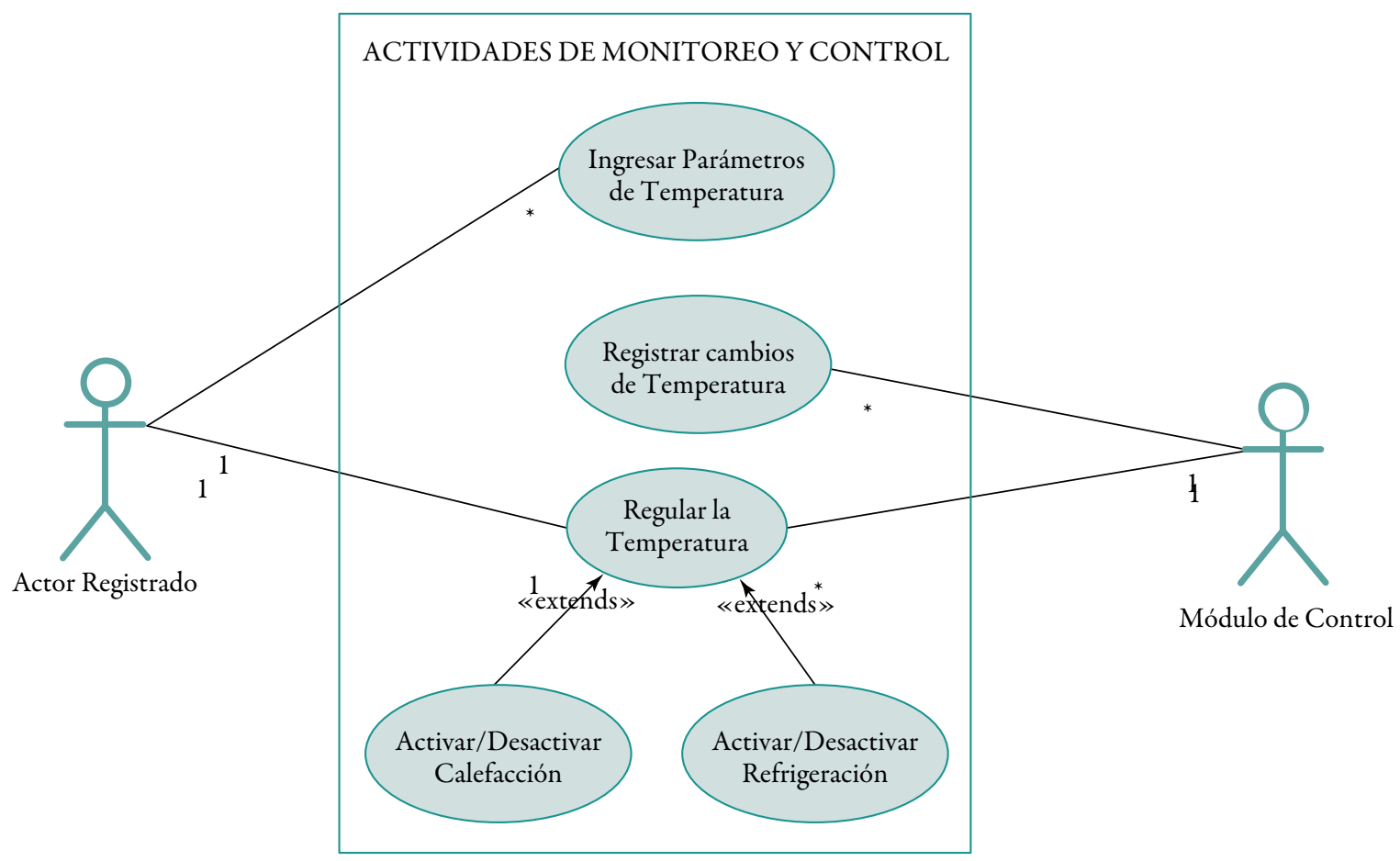

Figura 2. Monitoreo y control: diagrama de casos de uso. DCU-002.

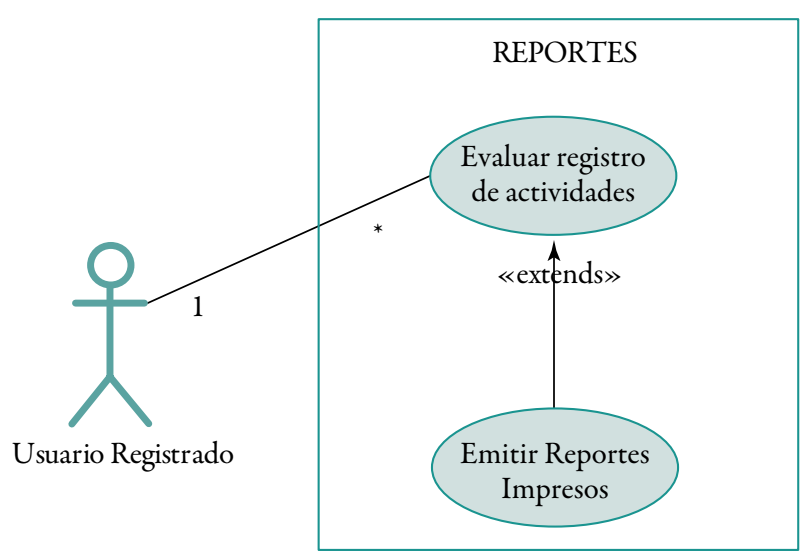

Figura 3. Caso de uso de reportes: DCU-003. 


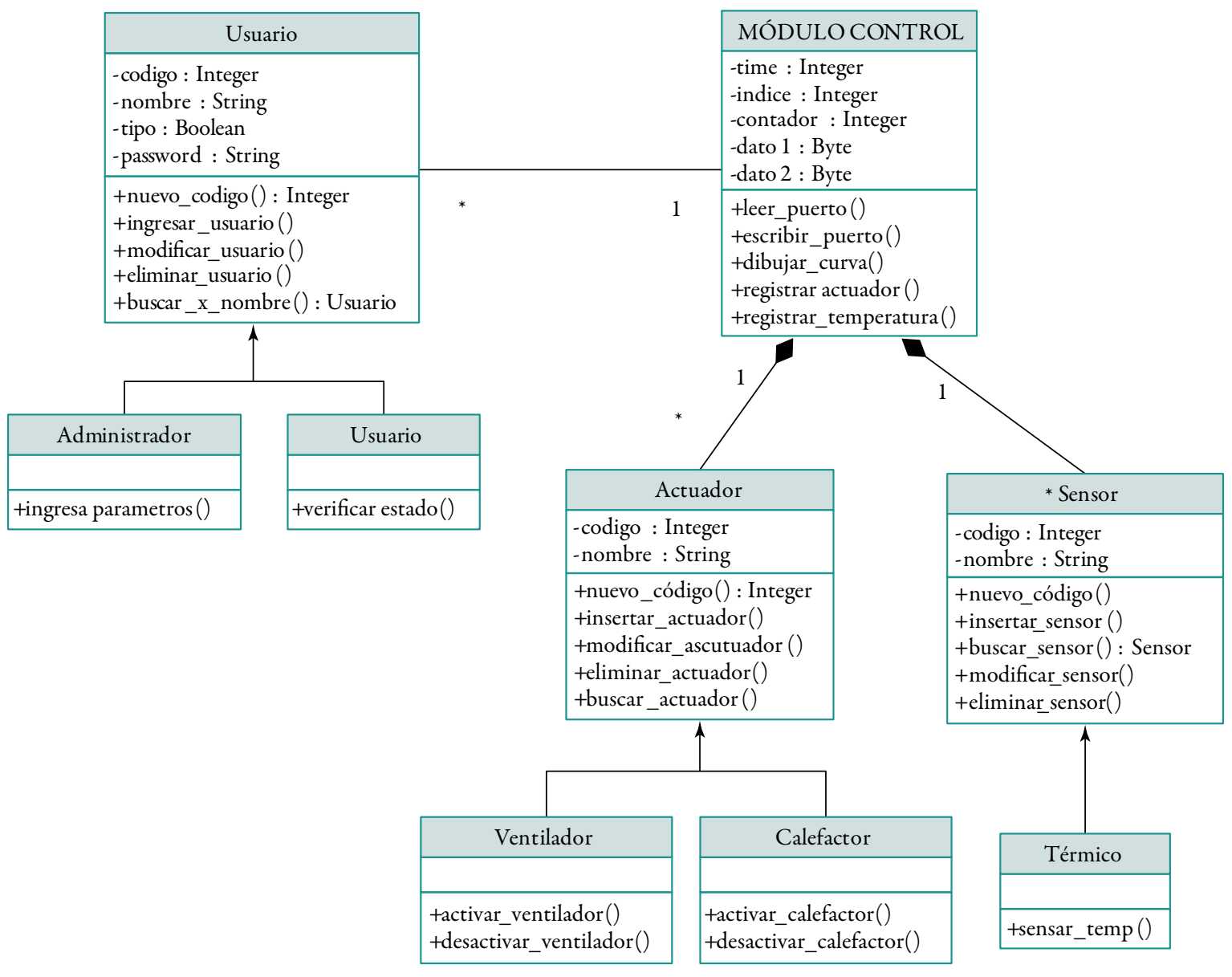

Figura 4. Diagrama de clases de diseño.

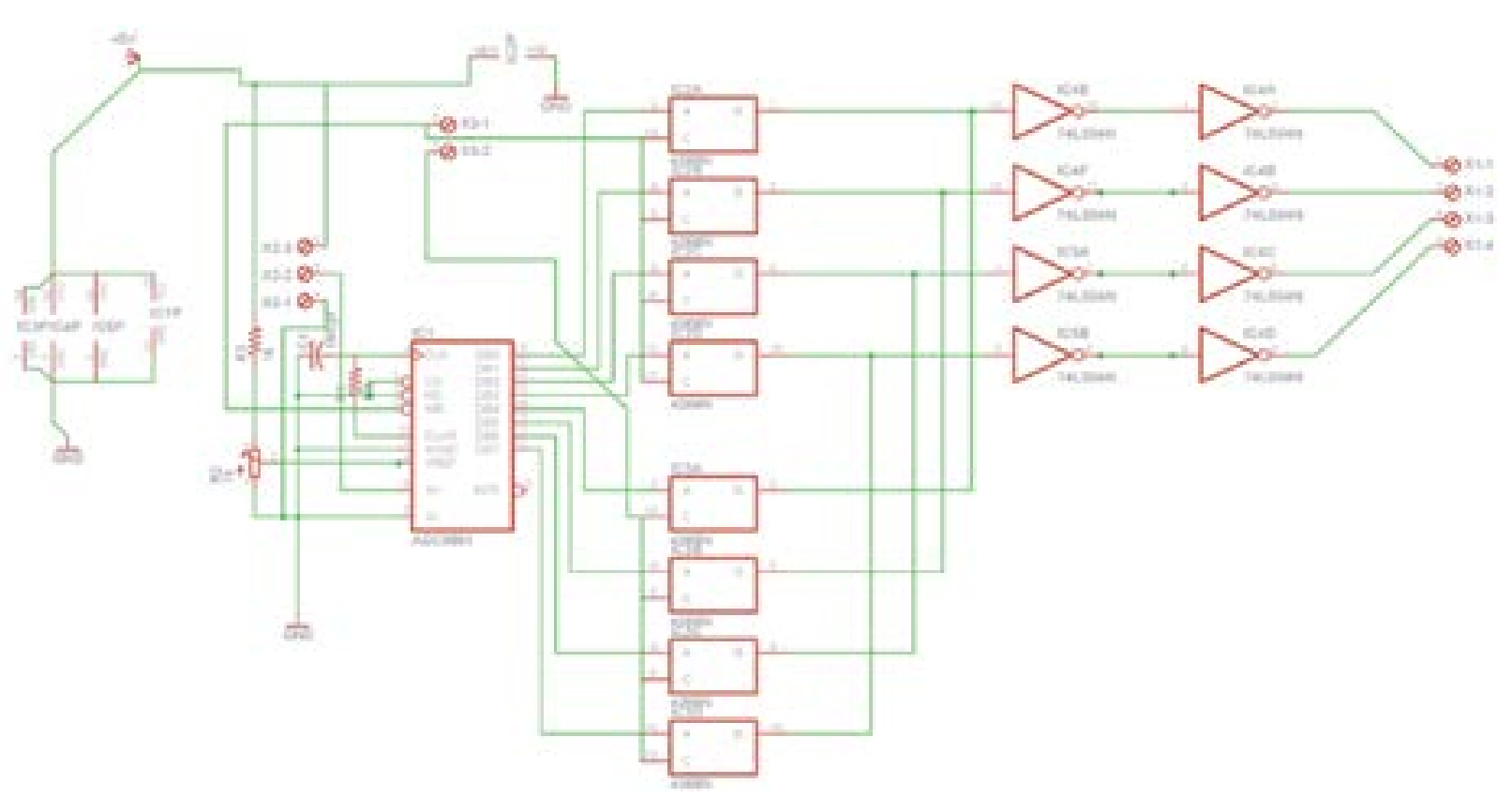

Figura 5. Diagrama esquemático del circuito de adquisición de datos. 


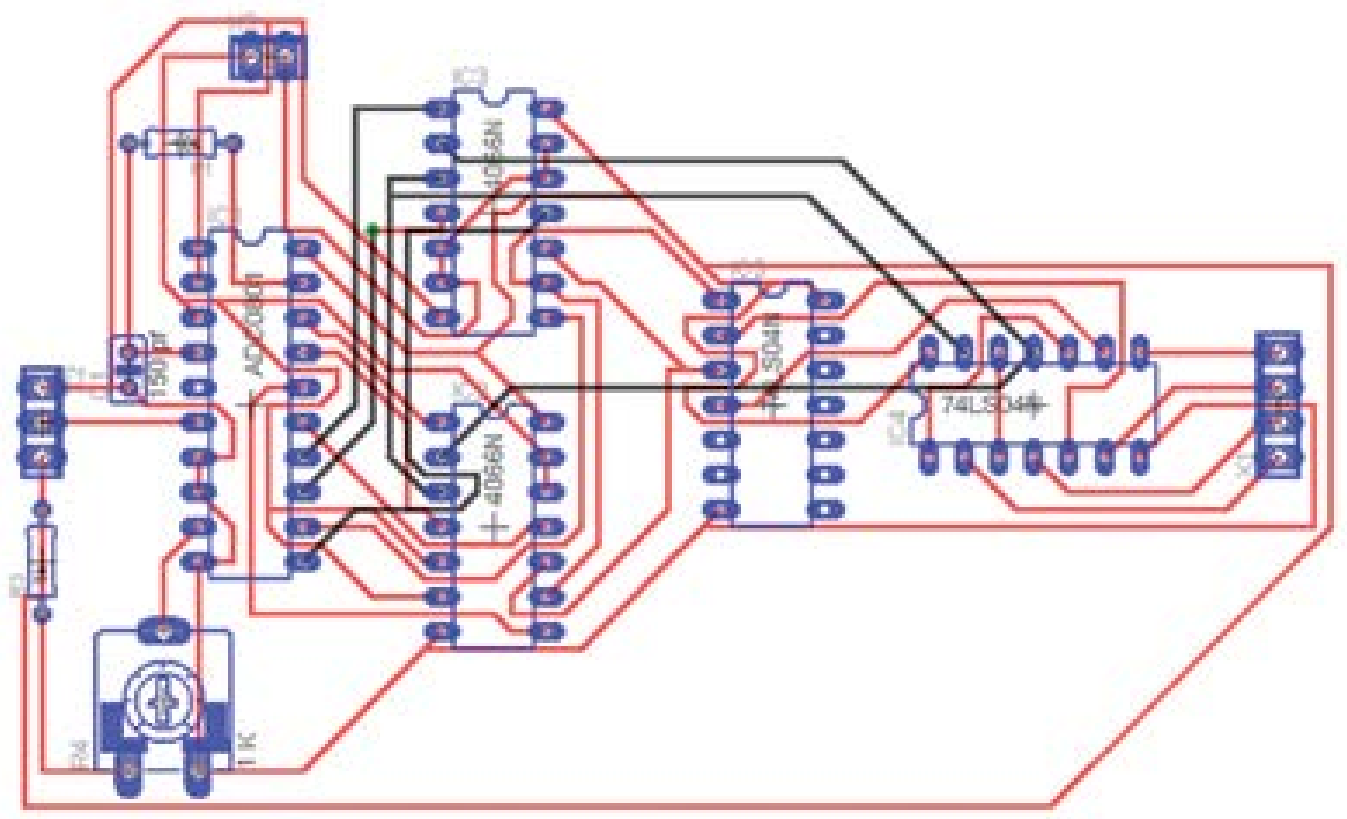

Figura 6. Pistas y dispositivos físicos de la tarjeta de adquisición

Circuito de fuerza del sistema de control ambiental

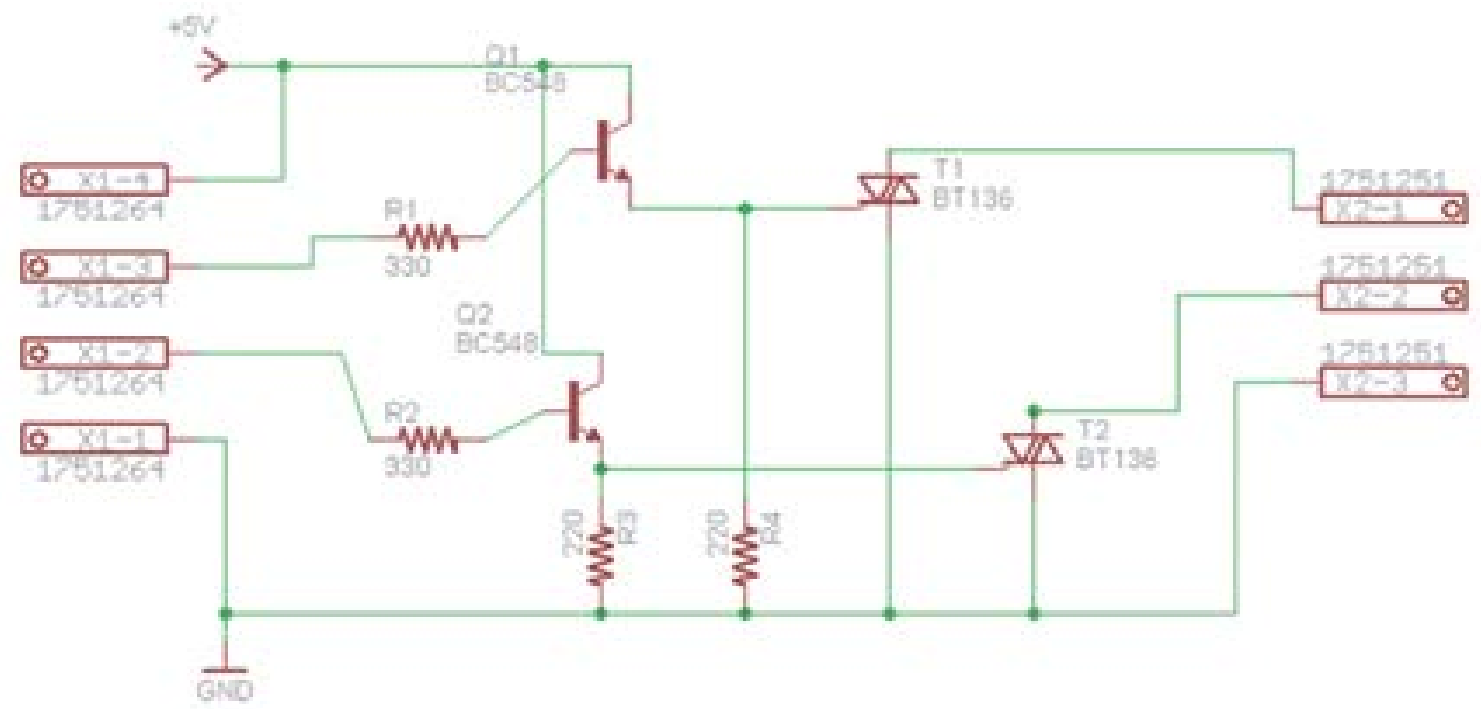

Figura 7. Diagrama esquemático del circuito de fuerza

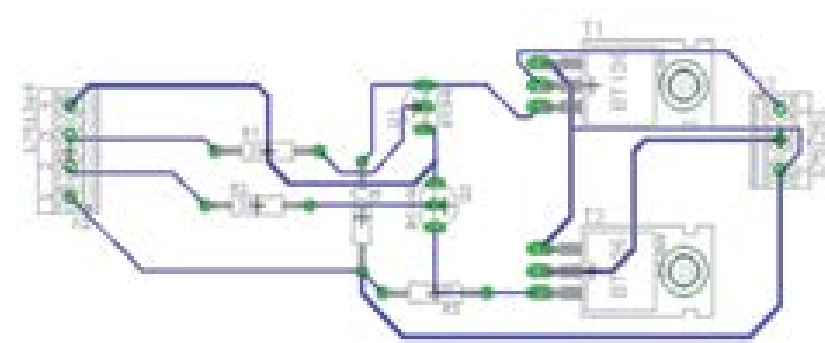

Figura 8. Diseño de pistas y componentes

Las figuras 5 a 8 representan los diagramas de los circuitos electrónicos utilizados para la elaboración de la tarjeta de adquisición de datos, es decir, la que convierte datos reales(por ejemplo, temperatura) en datos digitales, para ingresarlos luego a la PC y su posterior tratamiento y representación 


\section{Explotación de información}

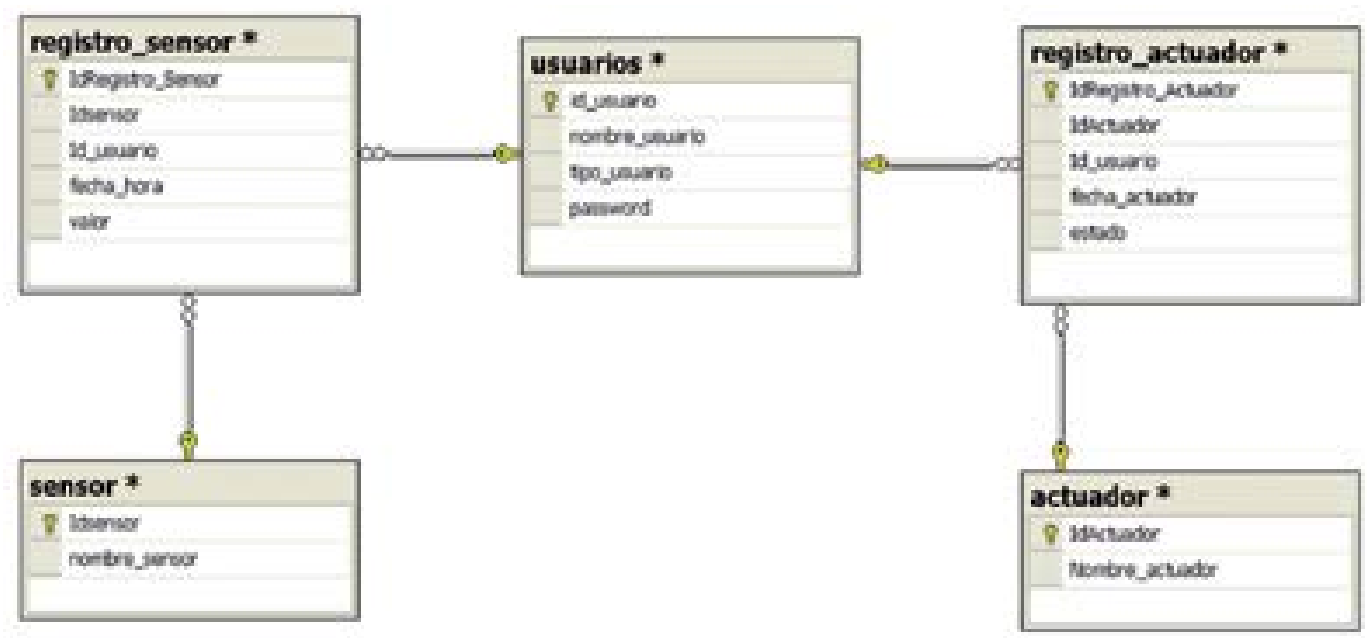

Figura 8. Diagrama de base de datos

Este diagrama representa la estructura de la base de datos diseñada para almacenar la información, a fin de explotar luego dichos datos, es decir, obtener la información requerida y necesaria para la toma de decisiones.

\section{Módulo de control del sistema}

Este módulo es el corazón de este trabajo de investigación, ya que es el encargado de sincronizar la tarjeta de adquisición de datos, recepción, tratamiento y representación de los mismos; también tiene la función de registro en forma automática de los valores de temperatura obtenidos, guardándolos en la base de datos en intervalos de tiempo que el usuario debe escoger, mostrando una gráfica en tiempo real lo cual representa la temperatura en función del tiempo. (Temperatura $\mathrm{T}$ en grados centígrados y tiempo $t$ en segundos.)

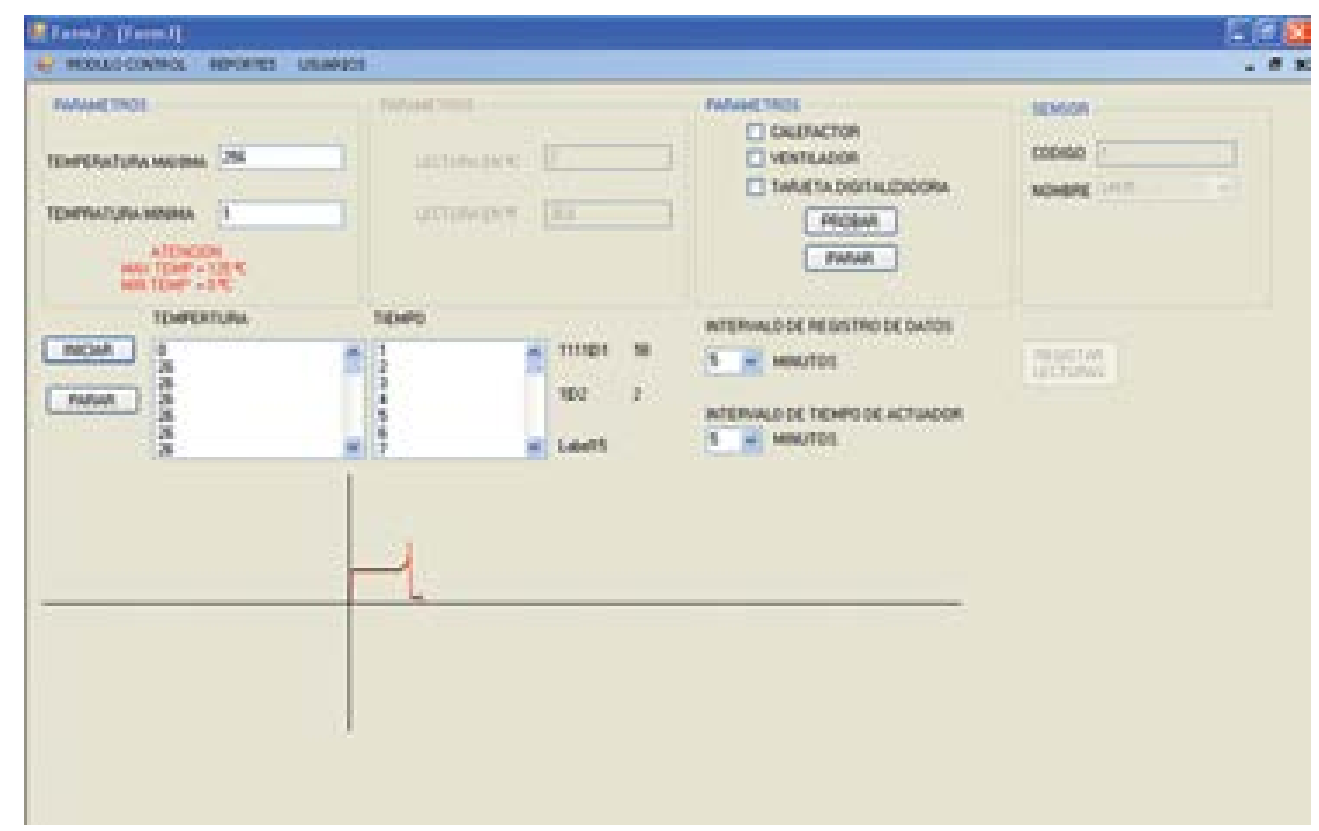

Figura 9 
Formulario de reportes

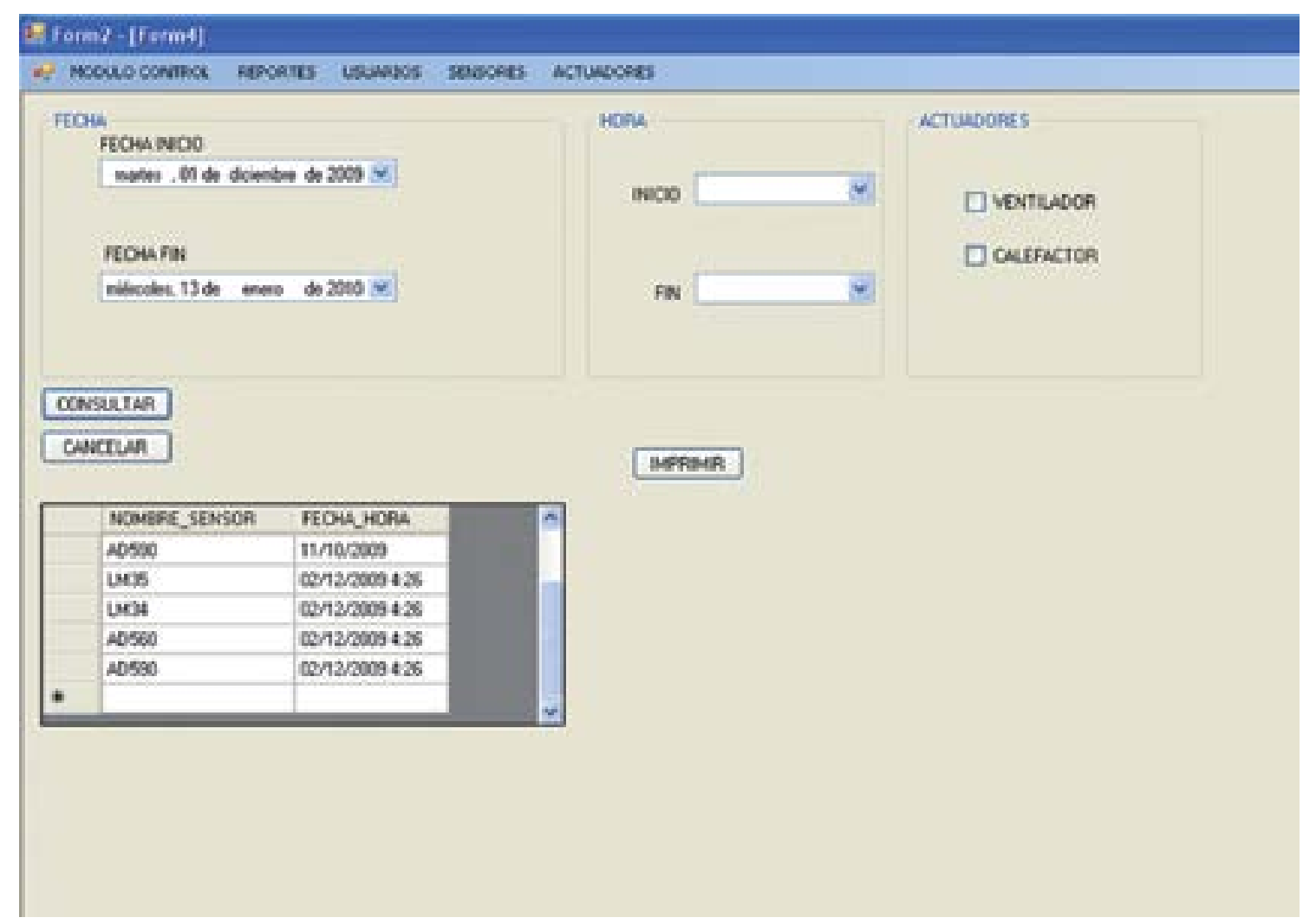

Figura 10. Formulario de consultas y reportes del sistema de control de temperatura

\section{Funcionamiento}

- Como se puede apreciar, en la figura 10, es posible hacer consultas de los registros de temperatura, mostrándonos el nombre del sensor y la fecha.

- Podemos delimitar una hora de inicio y una de fin, para verificar registros en un período de tiempo.

- Permite consultar por los actuadores, es decir consultas por fechas, así como por horas y por cada actuador específico ventilador y calefactor.

- Dicha parte del aplicativo permitirá conocer de manera rápida y completa el comportamiento y desempeño, tanto de los sensores como de los actuadores, que para nuestro caso son 2: ventilador y calefactor, encargados de subir y bajar la temperatura del ambiente cerrado respectivamente. 


\section{RESULTADOS}

Tabla 1. Resultado de las lecturas del puerto

\begin{tabular}{|c|c|c|c|c|}
\hline No PRUEBA & HORA & FECHA & VALOR To & $\begin{array}{l}\text { DATO INGRESADO } \\
\text { EN EL PUERTO H\&379 }\end{array}$ \\
\hline 1 & 0:00:00 & 10.11 .2009 & 28 & 11100 \\
\hline 2 & 0:00:00 & 12.02 .2009 & 29 & 11101 \\
\hline 3 & $4: 44: 12$ & 12.02 .2009 & 31 & 11111 \\
\hline 4 & $4: 26: 22$ & 12.04 .2009 & 33 & 100001 \\
\hline 5 & $15: 38: 17$ & 12.04 .2009 & 32 & 100000 \\
\hline 6 & $13: 01: 29$ & 12.04 .2009 & 33 & 100001 \\
\hline 7 & $15: 11: 01$ & 12.04.2009 & 30 & 11110 \\
\hline 8 & $4: 15: 59$ & 12.04 .2009 & 22 & 10110 \\
\hline 9 & $4: 16: 43$ & 12.04.2009 & 27 & 11011 \\
\hline 10 & $15: 21: 37$ & 12.04.2009 & 30 & 11110 \\
\hline 11 & $15: 24: 28$ & 12.04 .2009 & 31 & 11111 \\
\hline 12 & $15: 27: 13$ & 12.04 .2009 & 33 & 100001 \\
\hline 13 & $15: 28: 56$ & 12.04 .2009 & 32 & 100000 \\
\hline 14 & $15: 33: 57$ & 12.04 .2009 & 32 & 100000 \\
\hline 15 & $15: 33: 58$ & 12.04 .2009 & 35 & 11111 \\
\hline 16 & $16: 39: 08$ & 12.04 .2009 & 30 & 100001 \\
\hline 17 & $16: 41: 28$ & 12.04.2009 & 34 & 100001 \\
\hline 18 & $16: 44: 03$ & 12.04 .2009 & 33 & 100000 \\
\hline 19 & $16: 45: 47$ & 12.04.2009 & 32 & 100011 \\
\hline 20 & $16: 47: 15$ & 12.04 .2009 & 32 & 11110 \\
\hline 21 & $16: 47: 40$ & 12.04 .2009 & 31 & 100010 \\
\hline 22 & $16: 48: 22$ & 12.04 .2009 & 35 & 100001 \\
\hline 23 & $16: 49: 15$ & 12.04 .2009 & 32 & 100000 \\
\hline 24 & $16: 50: 22$ & 12.04 .2009 & 30 & 100000 \\
\hline 25 & $16: 52: 04$ & 12.04.2009 & 27 & 11011 \\
\hline 26 & $16: 52: 15$ & 12.04 .2009 & 29 & 11101 \\
\hline 27 & $16: 52: 29$ & 12.04 .2009 & 28 & 11100 \\
\hline 28 & $16: 52: 51$ & 12.04 .2009 & 30 & 11110 \\
\hline 29 & $17: 02: 55$ & 12.04 .2009 & 31 & 11111 \\
\hline 30 & 17:05:41 & 16.12 .2009 & 33 & 100001 \\
\hline
\end{tabular}

Elaboración propia

\section{CONCLUSIONES}

- Se logró controlar la temperatura en un critico estado en un ambiente cerrado mediante la digitalización de la temperatura y el uso de actuadores.

- Se construyó una tarjeta de adquisición de datos, así como una tarjeta de control de potencia para la activación de actuadores.

- El sistema fue probado en una PC Pentium III con rendimiento aceptable, estableciendo como requisitos mínimos para su funcionamiento, PIII de 500 Mhz, 256 Mb de RAM y tarjeta de video de $64 \mathrm{Mb}$. 
- El aplicativo nos ofrece el registro de las lecturas de temperatura, así como la activación de los actuadores en una $\mathrm{BD}$, lo cual permite análisis de los datos.

- Cambiando el tipo de sensor, se puede digitalizar otra magnitud real, como por ejemplo la humedad, la presión, etc.

\section{TRABAJOS FUTUROS}

Sistema de control automático, enfocándose a la domótica, así como la complementación de sistemas de rastreo con múltiples entradas, como Sistema de Monitoreo y Diagnóstico Clínico de Signos Vitales.

El uso de las TIC (Tecnologías de la Información y las Comunicaciones) en la vivienda genera nuevas aplicaciones $y$ tendencias basadas en la capacidad de proceso de información y en la integración y comunicación entre los equipos e instalaciones. Así concebida, una vivienda inteligente puede ofrecer una amplia gama de aplicaciones en áreas tales como:

- Seguridad.

- Gestión de la energía.

- Automatización de tareas domésticas.

- Formación, cultura y entretenimiento.

- Monitorización de la salud.

- Comunicación con servidores externos.

- Ocio y entretenimiento.

- Operación y mantenimiento de las instalaciones.

Como podemos apreciar, en todos estas áreas se pueden aplicar, complementar y proyectar investigaciones futuras.

\section{REFERENCIAS BIBLIOGRÁFICAS}

1. Malvino, Albert Paul.Principios de electrónica. Sexta edición. Editorial McGraw Hill.

2. Boylestad y Nachelsky. Electrónica. Teoría de circuitos. Sexta edición. Editorial Pearson Educación.

3. Tocci, Ronald. Sistemas digitales. Octava edición. Editorial Prentice Hall.

4. Canchucaja Vílchez, José del Carmen. Desarrollo de aplicaciones visual basic.net. Lima. Empresa Editora Macro, 2005.

5. Foxall, James D. Manual Visual Basic 2005. ANAYA Multimedia, octubre 2006.

6. Ángel Esteban. Desarrollo de aplicaciones para Internet con Asp .Net. Grupo editorial EIDOS.

\section{SITIOS WEB CONSULTADOS}

1. www.national.com

2. www.datasheets.com

3. www.yoreparo.com

4. www.electronicapablin.com

5. http://www.monografias.com/trabajos17/ sistemas-adquisicion-dato/sistemasadquisicion-dato.shtml

6. http://ambientesinteligentes.unizar.es/ index2.html

7. http://www.universia.es/btml_estatico/ portada/actualidad/noticia_actualidad/ param/noticia/hhgji.html

8. http://usuarios.lycos.es/charlytospage/ ADC0804_en_free_R.htm

9. www.philips.com 10/05/2009

10. http://www.datasheetcatalog.com/ datasheets_pdf/A/D/C/0/ADC0804.shtml 
11. www.casadomo.com 15/05/2009

12. http://www.casadomo.com/noticiasDetalle. asp $x ? c=14$ \& $m=21$ \&idm $=21$ \&pat $=20$ \&n $2=2$

13. www.universia.com $25 / 1 / 2005$
14. http://www.universia.es/btml_estático/ portada/actualidad/noticia_actualidad/ param/noticia/hhgji.html

15. Sobre un trabajo de Ing. Fernando Ventura Gutiérrez y la coordinación de Ing. Horacio D. Vallejo 16/05/2009bttp://www. webelectronica.com.ar/news $15 /$ nota09.htm 